\title{
Research on DSM Project Evaluation Model Based on AHP
}

\author{
Li Wang, Xiao-duan Wang and Qing Mi
}

Informatization Department, Hebei Academy of Governance, Shijiazhuang, 050000, China

\author{
Keywords: Demand side management (DSM); AHP; Comprehensive evaluation
}

\begin{abstract}
DSM project implementation includes a plurality of main power plants, power companies, users, etc. It not only need to consider the economic and social benefits, but also to ensure fair market complex market process. It is necessary to scientifically and comprehensively evaluate the effect of DSM project. The AHP based on the index scale is used in this paper to analyze and determine the weight of the various factors. In addition, the data pre-processing is implemented to form the evaluation set of various factors. Considering a variety of factors such as economic, environmental and social factor, the model to evaluate DSM project is established. A numerical example demonstrates that the proposed model is rational.
\end{abstract}

\section{Introduction}

Demand Side Management (DSM) is a comprehensive systems engineering. It is a overall planning which need each parties to business and require the returns together. Establishing a complete evaluation system for DSM project not only can improve the evaluation indicators of DSM in our country, fully tap the incentives of the parties involved and then promote the implementation of DSM programs in our country, but also has a guidance influence for the planning and implementation of DSM programs, and how to coordinate with practical guidance between participators.

Cost-benefit analysis method is widely used to assess the effectiveness of the implementation of DSM. Establishing a complete evaluation model need to consider three main aspects: Firstly, analyze and evaluate the factors involved, determine the weight of these issues based on the actual weight problem. Secondly, quantify the various factors in order to remove the difference in their properties so that they can be compared mathematically. Finally, considering practical issues, combining the weight of each factors mentioned above and its quantified value, the evaluation model is established to accurately describe the influence and relationship between these various factors [1-3].

\section{The determination of each factor's weight}

Analytic hierarchy process (AHP) is a combination of qualitative and quantitative, systematic, hierarchical analysis problems method. It makes the layers hierarchical through the people's thought processes, compare relevant factors and intervening layer by layer test to compare the results are reasonable, so as to analyze the decision-making to provide a more convincing quantitative basis.

This paper divides the factor in the DSM into three aspects including economic factors, social and environmental factors. The AHP method is defined as follows [4]:

(1) Target level: The total evaluation value.

(2) Criterion level: It includes economic factors, social and environmental factors.

(3) Methods level: The economic factor includes the cost-benefit analysis for power plants, power company and participating users. The social factor includes the fairness and the cost-benefit analysis for non- participating users. The environmental factor includes the cost-benefit analysis for environment.

Consider different factors, the judgment matrix $A$ with the form of scaling exponent is constructed as follows: 


$$
A=\left[\begin{array}{ccccc}
a^{0} & a^{1} & a^{4} & a^{5} & a^{7} \\
a^{-1} & a^{0} & a^{4} & a^{5} & a^{7} \\
a^{-4} & a^{-4} & a^{0} & a^{1} & a^{6} \\
a^{-5} & a^{-5} & a^{-1} & a^{0} & a^{5} \\
a^{-7} & a^{-7} & a^{-6} & a^{-5} & a^{0}
\end{array}\right] .
$$

Among them, each row from left to right ( or top to bottom ) respectively represents power companies, power plants, participating users, fairness and non-participating users. The value of $a$ in the matrix is taken as 1.618 .

Because the judgment matrix given by decision makers always can not satisfy the consistency. Thus, it is necessary to make judgment matrix consistency test. When the result of consistency test is poor, it need to correct the matrix. In order to meet the requirements of complete consistency, the evaluation judgment matrix need to go through the following tests:

$$
C I=\frac{\lambda_{\max }-n}{n-1}
$$

Where $\lambda_{\max }$ is the largest eigenvalue in the judgment matrix.

If the value of $C I$ is larger, the consistency of judgment matrix is more poor. In practice, the specific approach is comparing with the average consistent index $R I$, namely:

$$
C R=\frac{C I}{R I}
$$

Where $C R$ represents the consistency ratio of the judgment matrix. When $C R$ is below 0.1 , the judgment matrix satisfies .consistency. Otherwise, it need to be corrected.

For the judgment matrix in this paper, the $C R$ equals 0.0523 , which satisfies the consistency. The eigenvectors of $\lambda_{\max }$ is normalized and the weight vector is obtained.

$$
W=\left(W_{c o m}, W_{G}, W_{c u s}, W_{r}, W_{p}\right) .
$$

Where $W_{\text {com }}, W_{G}, W_{\text {cus }}, W_{r}, W_{p}$ are respectively the weight value of power companies, power plants, participating users, fairness and non-participating users. The calculation result is shown as follows:

$$
W=\left(\begin{array}{lllll}
0.394 & 0.349 & 0.131 & 0.096 & 0.031
\end{array}\right) .
$$

In the implementation process of DSM, the environmental benefits are required by sacrificing the economic interests of power companies, power plants and participating users. Thus, the its weight can be thought as the average of weights of power companies, power plants and participating users, which is shown as follows:

$$
W_{E}=\frac{W_{c o m}+W_{G}+W_{c u s}}{3}=0.291
$$

\section{The Determination of evaluation set}

Through analyzing and summarizing the costs and benefits functions of each factor, the evaluation set is formed after pre-processing the cost-effectiveness data.

Firstly, no -dimension processing is implemented as follows. 
$R_{i}=\frac{B_{i}}{C_{i}},(i=1,2,3,4,5)$

Where $B_{i}$ is .effectiveness value represents of the $i_{\text {th factor. }} C_{i}$ represents the cost value of the $i_{\text {th factor. }} R_{i}$ represents the original cost-benefit ratio of the $i_{\text {th factor. }}$

Then, because the importance of various factors for DSM is different, the cost-effective ratio of the economic factors need to be normalized. There these three economic factors can be compare mathematically, which can form the fairness factor.

$$
\begin{aligned}
& C_{o}=\frac{R_{1}}{W_{c o m}} . \\
& C_{e}=\frac{R_{2}}{W_{G}} . \\
& C_{u}=\frac{R_{3}}{W_{c u s}} .
\end{aligned}
$$

Where $C_{o}, C_{e}, C_{u}$ are respectively the cost-benefit ratio of power companies, power plants and participating users.

In summary, the evaluation set of each factor is formed as follows:

$$
M=\left(C_{o}, C_{e}, C_{u}, \Gamma, E, P\right) \text {. }
$$

$$
E=R_{5} \text {. }
$$

$$
P=R_{4} \text {. }
$$

Where $C_{o}, C_{e}, C_{u}, E, P$ are respectively the quantitative evaluation value of power companies, power plants, participating users, environmental benefits and non-participating users. $\Gamma$ is the fairness factor.

\section{The establishment of DSM project evaluation model}

\section{Economic factors.}

In DSM, the economic factor is due to the following three sub- factors: cost-effective power plants, electric power company's cost -effectiveness and cost-effectiveness of power user. The economic factors influence model is constructed as follow:

$$
\begin{aligned}
& E_{c o}=\left(W_{G} C_{e}+W_{c o m} C_{o}+W_{c u s} C_{u}\right) \Psi_{G} \Psi_{c o m} \Psi_{c u s} . \\
& \Psi_{G}= \begin{cases}1 & C_{e} \geq \varepsilon \\
\delta C_{e} & C_{e}<\varepsilon .\end{cases} \\
& \Psi_{\text {com }}= \begin{cases}1 & C_{o} \geq \varepsilon \\
\delta C_{o} & C_{o}<\varepsilon\end{cases} \\
& \Psi_{\text {cus }}= \begin{cases}1 & C_{u} \geq \varepsilon \\
\delta C_{u} & C_{u}<\varepsilon .\end{cases}
\end{aligned}
$$


Where $E_{c o}$ represents economic value. $\Psi_{G}, \Psi_{\text {com }}, \Psi_{\text {cus }}$ respectively represents the influence function from cost-effectiveness to economic value of power companies, power plants and participating users. $\varepsilon$ is the threshold of influence function. $\delta$ is the scaling factor of penalty factor.

\section{Environmental benefits.}

An important role for implementing DSM project is to increase energy efficiency and reduce pollution from power plants on the environment. Therefore, it is necessary consider the environmental benefits. When the quantization value of environmental benefits is less than the threshold value, it will appear in the model with the probability below 1, which make the overall effectiveness evaluation be smaller. Its influence function is constructed as follow:

$\Psi_{E}= \begin{cases}1 & \xi<E<\eta \\ 1+E W_{e} & E \geq \eta \\ \gamma E & E<\xi\end{cases}$

Where $\Psi_{E}$ represents influence function from environmental benefits to the total evaluation model. $E$ represents the quantized value of environmental benefits.

\section{Social factors}

The fairness is introduced to reflect the benefits distribution among power companies, power plants and participating users. And the fairness value will be quantified as follows: $\Gamma=\frac{\left(C_{e}-A V E\right)^{2}+\left(C_{o}-A V E\right)^{2}+\left(C_{u}-A V E\right)^{2}}{3\left(C_{e}+C_{o}+C_{u}\right)}$

$A V E=\frac{C_{e}+C_{o}+C_{u}}{3}$

Where $\Gamma$ is the value of fairness.

The influence function is defined as follows:

$\Psi_{\Gamma}=\left\{\begin{array}{cc}1 & \Gamma<\lambda \\ 1-\Gamma W_{\Gamma} & \Gamma \geq \lambda\end{array}\right.$.

Where $\lambda$ is the threshold of fairness factor.

Non-participating user is the indirect object of DSM implementation, and its benefits comes from avoiding cost of from the power sector. Its influence function is defined as follow:

$\Psi_{\text {Ncou }}=\left\{\begin{array}{cl}1 & 0<P<\mu \\ \sqrt{1+\left(P \cdot W_{p}\right)^{2}} & P \geq \mu \\ \beta P & P \leq \theta\end{array}\right.$.

Where $P$ represents cost-benefit ratio of non-participating users. $\beta$ is the scale factor to describe the impact of the on-participating users in the evaluation model.

Taking these economic factors, environmental factors and social factors, the DSM evaluation model is established as follows:

$$
E_{N W}=K \cdot E_{e o} \cdot \Psi_{\Gamma} \cdot \Psi_{E} \cdot \Psi_{N c o u}
$$


Where $E_{N W}$ is the total evaluation value of DSM. It is an efficiency target, the greater its value, then evaluation result is better. $K$ is a amplification factor in order to facilitate the comparison of the evaluation values.

\section{Specific example and results analysis}

Applying the proposed method to a certain city for implementing the cost-effectiveness analysis of TOU price. In addition, the cost-effectiveness ratio of the non-participating users is regarded as 1 . The original data and cost-effectiveness ratio are shown in Table 1.

Table 1: Cost-effectiveness analysis

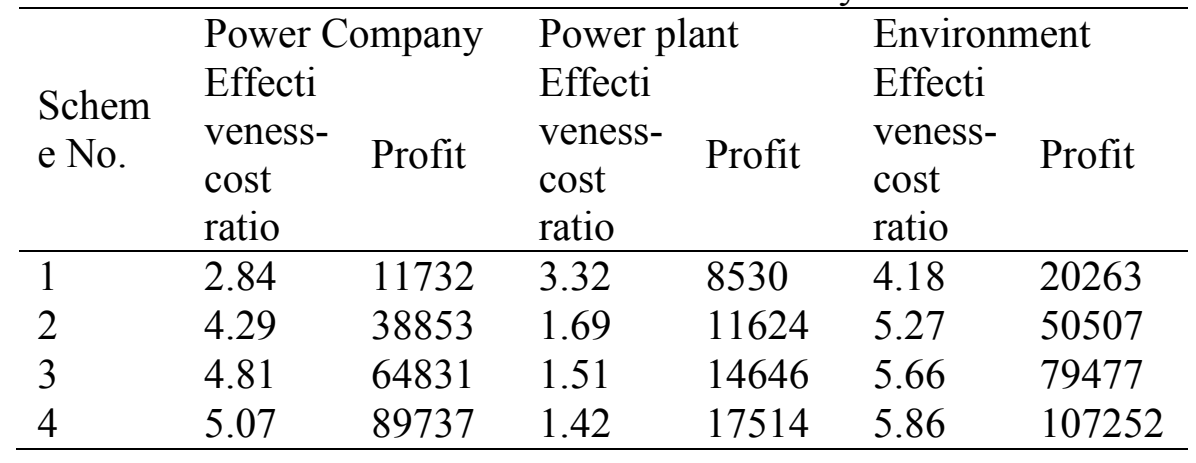

The above data are put into the evaluation model, and the comprehensive evaluation value of each scheme can be obtained. For comparison convenience, another four schemes are implemented in the same model, which are shown in Table 2.

Table 2: Comprehensive evaluation results

\begin{tabular}{llllllll}
\hline Scheme & $C_{o}$ & $C_{e}$ & $C_{u}$ & $\Gamma$ & $E$ & $P$ & $R_{v a}$ \\
\hline 1 & 2.84 & 3.32 & 1 & 0.42 & 4.18 & 1 & 47.63 \\
2 & 4.29 & 1.69 & 1 & 0.26 & 5.27 & 1 & 49.03 \\
3 & 4.81 & 1.51 & 1 & 0.43 & 5.66 & 1 & 51.78 \\
4 & 5.08 & 1.42 & 1 & 0.53 & 5.86 & 1 & 51.39 \\
5 & 5.07 & 1.42 & 1 & - & 5.86 & 1 & 60.81 \\
6 & 5.21 & 1.31 & 1 & - & 5.86 & 1 & 60.89 \\
7 & 6.11 & 2.21 & 1 & 0.56 & 5.86 & 1 & 63.25 \\
8 & 6.11 & 2.21 & 1 & 0.56 & 5.86 & 0.8 & 60.72 \\
\hline
\end{tabular}

As can be seen from Table 2, No.7 scheme obtains the highest

comprehensive evaluation value due to its high economic returns. Only DSM continuously improve the overall economic benefits, ensure a reasonable allocation in the economic body, take into account environmental and social impacts, can make the implementation of DSM more efficient.

\section{Conclusions}

DSM project involves various aspects of cost-benefit analysis among power plants, power companies, and even power users and society. The analysis of one aspect can not be sole criterion for DSM project implementation. This paper applies qualitative analysis and quantitative analysis, establish an evaluation model and uses AHP based on index to the weight of economic and social factors, achieve a comprehensive evaluation of DSM. A numerical example demonstrates that the proposed model is rational. 


\section{References}

[1] Saurabh Gupta, Tanushree Bhattacharya. DSM Power Plant in India, Renewable and Sustainable Energy Reviews. 21 (6), pp. 537-547,2013.

[2] Asal Hamarneh. DSM proposes new roads to weight reduction. Reinforced Plastics. 58 (11), pp. 19-21,2014.

[3] A.G. Ramm. DSM for general nonlinear equations. Applied Mathematics Letters. 25 (15), pp. 2009-2014,2012.

[4] F. Lolli, A. Ishizaka, R. Gamberini. New AHP-based approaches for multi-criteria inventory classification. International Journal of Production Economics. 156 (7), pp. 62-74,2014.

[5] Navjot Kaur, Shailesh Kumar, Shanmugam Mayilraj. Genome sequencing and annotation of Amycolatopsis vancoresmycina strain DSM 44592T. Genomics Data. 2, pp. 16-17, 2014.

[6] Gertrud Sofie Hafstad, Grete Dyb, Tine K. Jensen, Alan M. Steinberg, Robert S. Pynoos. PTSD prevalence and symptom structure of DSM-5 criteria in adolescents and young adults surviving the 2011 shooting in Norway. Journal of Affective Disorders. 169, pp. 40-46, 2014.

[7] J. Christopher Fowler, Carla Sharp, Allison Kalpakci, Alok Madan, Joshua Clapp, Jon G. Allen, B. Christopher Frueh, John M. Oldham. A dimensional approach to assessing personality functioning: examining personality trait domains utilizing DSM-IV personality disorder criteria. Comprehensive Psychiatry. 56, pp. 75-84, 2015.

[8] Claudia Carmassi, Carlo Antonio Bertelloni, Gabriele Massimetti, Mario Miniati, Paolo Stratta, Alessandro Rossi, Liliana Dell'Osso. Impact of DSM-5 PTSD and gender on impaired eating behaviors in 512 Italian earthquake survivors. Psychiatry Research. 225(1 - 2), pp.64-69, 2015.

[9] Armando Calabrese, Roberta Costa, Tamara Menichini. Using Fuzzy AHP to manage Intellectual Capital assets: An application to the ICT service industry. Expert Systems with Applications. 40(9), pp.3747-3755, 2013.

[10] Reza Eslamipoor, Abbas Sepehriar. Firm relocation as a potential solution for environment improvement using a SWOT-AHP hybrid method. Process Safety and Environmental Protection. 92(3), pp. 269-276, 2014. 